\title{
DELIVERY OF ANTIMICROBIAL DRUGS ACROSS INFLAMMATORY MEMBRANE IN RABBITS
}

\author{
By ROBERT McCUNE \\ (From the Cornell University Medical College, New York, N. Y.)
}

(Submitted for publication September 8, 1959; accepted February 4, 1960)

An important criterion of the efficacy of a drug is its ability to traverse barriers in the animal body. Potential barriers may be either physiological, such as the lining of the body compartments and the blood-brain barrier, or those induced by disease. The latter afford an important test for the evaluation of antimicrobial agents. In a general sense, most in vitro tests put to new drugs are performed under optimal physical conditions, as of temperature and $\mathrm{pH}$, or are pharmacological experiments performed in the healthy body. There is an obvious interest in making test observations under conditions which simulate disease states and, in the case of antimicrobial drugs, inflammations.

Abscess walls, the vegetations of subacute bacterial endocarditis, exudates, and necrotic material are classic examples of host reactions to parasitism that conceivably might impede the transport of drug to invading microorganisms. For the most part, the judgment concerning a drug's ability or inability to penetrate into certain types of lesions is derived from the empirical clinical evidence that it is able or unable to control infections that are characterized by these types of lesions. In considering the many factors and uncontrollable variables involved in any host-drug-parasite relationship, it becomes apparent that this reasoning is superficial at best. The difficulty of making precise and pertinent measurements in patients suggests a need for experimental approaches to studies of the transport of antimicrobial substances across inflammatory barriers.

Considerable evidence indicates that foreign particles tend to localize in areas of inflammation in experimental animals (1-4). Markham and Florey have made observations on the penetration of carbon particles insicle lung tubercles (5). In the course of experiments concerned with the filtrating and dialyzing properties of a thin fibrin meml)rane constructed from pooled human plasma, it was observed that large blood proteins were unable to penetrate this barrier, whereas such substances as trypan blue, gentian violet, and staphylococcal and diphtheria toxins did penetrate it (6). Poor penetrating qualities of sulfanilamide, sulfapyridine, gentian violet, and merthiolate were observed in experiments involving fibrin clots and fibrin platelet membranes made from dog blood (7).

In experiments with fibrin-agar plates, it was observed that penicillin readily diffused into both fibrin and agar, whereas sulfathiozole and sulfadiazine diffused into agar but not fibrin (8). In studying the diffusion properties of penicillin with a membrane constructed by combining crystallized fibrinogen and pure thrombin in solution, it was noted that these characteristics varied with the concentration of penicillin used and the thickness of the membrane (9). Weinstein, Daikos and Perrin inserted fibrin clots into subcutaneous tissues of rabbits, administered intramuscularly various doses of penicillin, removed the clots and obtained blood samples over a 12 hour period (10). The results of analysis of the clots and sera demonstrated that the height and persistence of penicillin concentrations in the clots were proportional to the dosage of the penicillin administered. When localized aseptic inflammatory reactions were produced with turpentine in rabbits, good penicillin concentration levels were observed in the exudate within one-half hour after intramuscular administration of the drug (11). As was demonstrated in the studies with subcutaneous fibrin clots in rabbits, it again was observed that the height of the penicillin concentrations and the persistence of detectable amounts varied directly with the administered penicillin dosage.

A method was devised for localization of bacterial populations in unenriched agar discs which were successfully maintained in the peritoneal cavities of animal hosts for varying periods of time (12). As the bacteria were dependent for 
growth upon nutritive substances from the surrounding tissue fluids, they were thus able to exist in a state more closely resembling true parasitism than is possible in vitro. The tissue reaction to agar discs in the peritoneal cavity closely resembled a foreign body reaction in that a membrane formed around it. It is the purpose of this investigation to correlate the histological development of the membrane with the ability of penicillin and streptomycin to penetrate this structure in vivo.

\section{MATERIALS AND METHODS}

Preparation of agar discs. Five $\mathrm{ml}$ of 3 per cent unenriched agar was poured under sterile conditions into clean aluminum cylindrical molds $7 \mathrm{~mm}$ high with an inside diameter of $22 \mathrm{~mm}$, which were set in petri dishes resting on a cold surface; the agar was allowed to solidify.

Rabbits. Twelve male albino rabbits, average weight $3.0 \mathrm{~kg}$, were used.

Drug administration. Crystalline penicillin G was administered intravenously, $12.5 \mathrm{mg}$ per $\mathrm{kg}$. Streptomycin sulfate was administered intravenously, $60 \mathrm{mg}$ per $\mathrm{kg}$.

Distribution of drug in vivo. Rabbits were weighed and anesthetized with intravenous sodium pentobarbital. Six to eight 5-ml agar discs were introduced into the peritoneal cavity with care to ensure the contact of the flat surfaces of the agar discs with the peritoneum and to prevent apposition of the discs with each other. In one set of experiments after all of the discs had been inserted, the animals received an intravenous dose of penicillin. Specimens of blood were obtained by intracardiac puncture, and agar discs were removed at various intervals during the 24 hour period after drug administration. After the 6 hour specimens had been obtained the wounds were closed and the animals were allowed to regain consciousness. The animals were reanesthetized and new incisions were made at 24 hours. In order to prevent dehydration, the animals received by gavage $50 \mathrm{ml}$ of 10 per cent glucose in 1 per cent saline at 4 and at 24 hours after the start of the experiment.

In another set of studies, blood samples and 1 or 2 discs were removed at various intervals up to 28 days after implantation. In these studies, penicillin or streptomycin was administered intravenously 2 hours before removal of the discs and blood sample. When two discs were removed in the study, one was prepared for microscopic examination. The other disc and blood sample were assayed for antimicrobial drug content. After removal of the discs the animal was allowed to recover to be used at a subsequent study interval. The formed membranes surrounding the removed discs were carefully scrutinized to be assured that they were intact.

Assay for antimicrobial drug content. The blood was allowed to clot for 1 hour at $4^{\circ} \mathrm{C}$ and the serum was removed after centrifugation. After removal of the formed membranes the discs were diced with a sterile spatula and the resultant mass transferred to large test tubes containing an equal volume of broth. After vigorous shaking the tubes were incubated at $37^{\circ} \mathrm{C}$ for 1 hour to permit equilibration of drug. Microbiological assay was carried out by means of a fractional dilution technique capable of detecting smaller changes in drug concentrations than the conventional twofold serial dilution method (13). In the case of penicillin, almost exactly equal distribution of drug between agar and broth was achieved in this in vitro system. For streptomycin, however, the concentrations in the broth which had been exposed to agar containing drug were only 50 per cent of those that would have obtained had equal distribution of the drug between agar and broth occurred. This yielded factors of 2 for penicillin and 4 for streptomycin, respectively, in determining the original concentration of these drugs in the agar by this method. Standard solutions of drug in broth were assayed concurrently with the unknown specimens.

\section{RESULTS}

\section{Correlation of histopathological development of} membrane and ability of penicillin to penetrate into agar. Six to seven agar discs were inserted into the peritoneal cavity of each of three rabbits. Two discs were aseptically removed and a blood sample was obtained alternately from one of the three animals at $1,4,8,12,21$ and 28 days after implantation. The discs and blood specimens were obtained two hours following the intravenous administration of penicillin at a dosage of $12.5 \mathrm{mg}$ per $\mathrm{kg}$. One of the two discs removed was prepared for histological study of the surrounding membrane, whereas the other disc and the blood sample were assayed for penicillin content.

From the standpoint of gross appearance, the membranes that formed around the agar discs progressed from a thin, filmy, fragile structure 24 hours after implantation to a tough, durable, fairly inelastic 1 to $2 \mathrm{~mm}$ thick tissue at 28 days. After 21 days in vivo, the discs had usually become fixed by a band of fibrous tissue to some organ or to the lining wall of the peritoneal cavity.

A histological review revealed that 24 hours after implantation the disc membrane consisted of a thin fibrin layer containing an abundant collection of polymorphonuclear leukocytes. By 4 days the fibrin layer had become denser and thicker. The cellular elements still consisted mostly of polymorphonuclear leukocytes which 


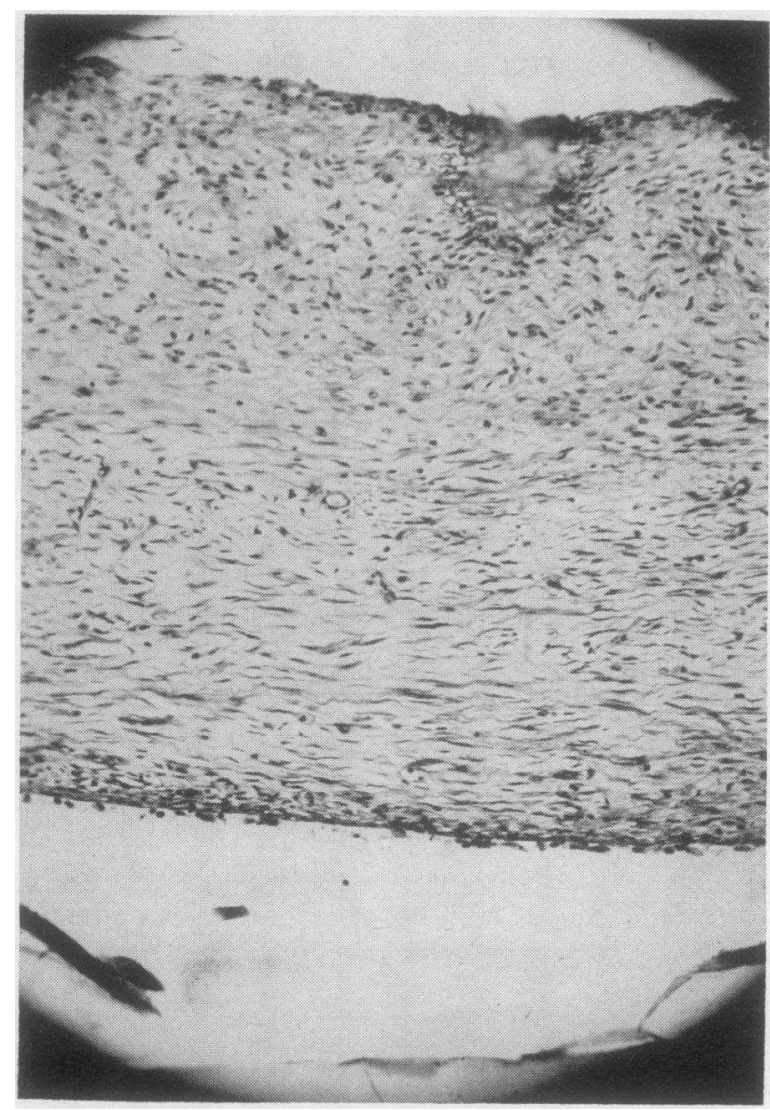

Fig. 1. Photomicrograph of Fibrous membrane SURROUNDING AGAR DISC REMOVED 28 DAYS AFTER IMPLANTATION IN PERITONEAL CAVITY OF RABBIT. exhibited signs of aging. Within the disc there were many round cells, and near the outer surface of the membrane there were a few having the appearance of mesothelial cells. By 8 days the bulk of the membrane consisted mainly of a loose, fibrinous structure containing scattered fibrocytes and fibroblasts. A moderate number of polymorphonuclear leukocytes and lymphocytes was concentrated near the agar, or inner, surface of the membrane. The polymorphonuclear leukocytes had undergone considerable degeneration with much evidence of karyhorrhexis and pyknosis. Giant cells were scattered throughout. The mesothelial cells near the outer border of the membrane had become flattened and elongated, suggesting that they were undergoing a transition to fibrocytes. By 13 days after insertion into the peritoneal cavity, there was a definite polarization of the fibrillar structure of the membranes with scattered fibrocytes and fibroblasts throughout, giving the appearance of a very young collagenous structure. Occasional bits of fibrinous material were still present. There was a small collection of round cells near the inner border of the membrane, with only rare nests of degenerating polymorphonuclear leukocytes. At 21 days the fibrous tissue was more mature. Remaining elements of fibrin were only rarely seen, the cellular elements were

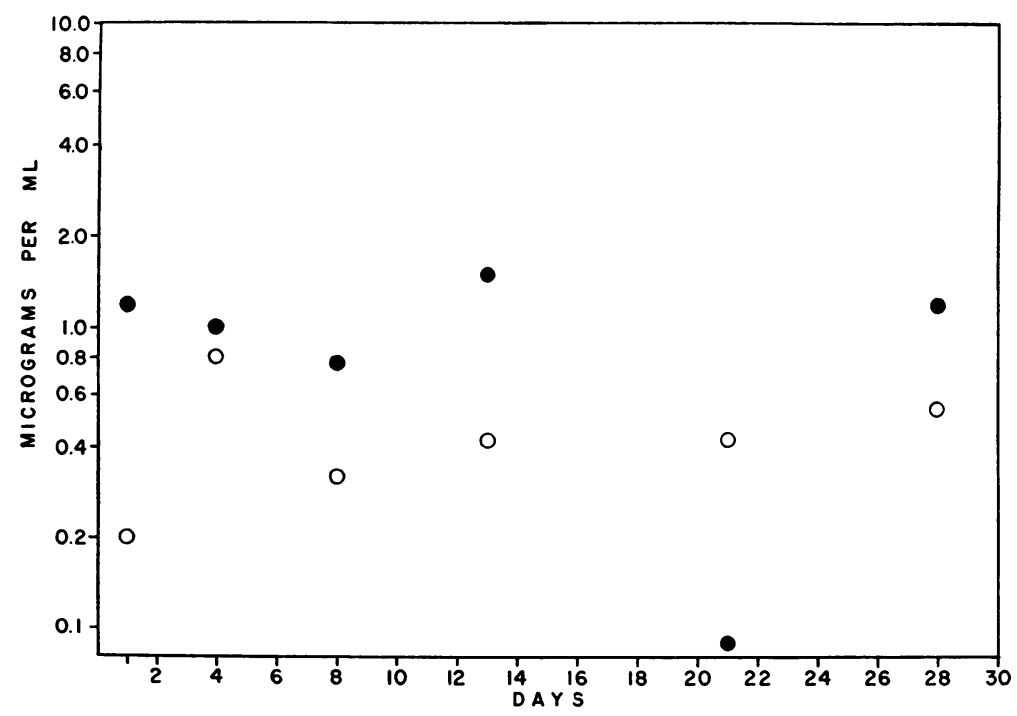

Fig. 2. Distribution of PENicillin between AGar discs and SERUm at VARIED TIMES AFTER IMPLANTATION OF AGAR IN PERITONEAL CAVITY OF RABBITs. Penicillin injected two hours before removal of discs; $\bigcirc$ serum concentration; agar concentration. 


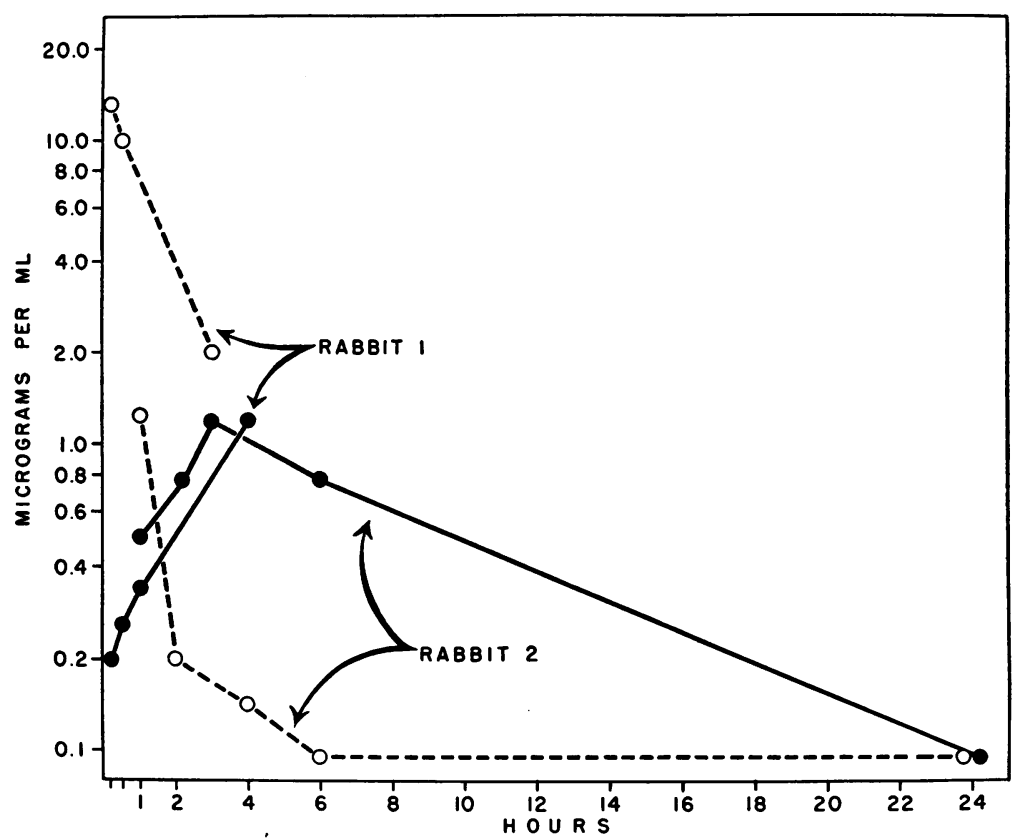

Fig. 3. Distribution of PENICILlin BetweEn AGAR DisCS AND SERUM AT ONE DAY AFTER IMPLANTATION OF DISCS IN PERITONEAL CAVITY OF RABBITS. Penicillin injected two hours before removal of discs; - agar concentration; $O$ serum concentration. For technical reasons two animals were needed to complete the experiment.

mostly fibroblasts, occasional giant cells could still be seen and evidence of early vascularization was observed. By 28 days after implantation in the peritoneal cavity, the membrane surrounding the agar disc had become a fairly thick layer of fibrous tissues with a moderate amount of early vascularization (Figure 1). The collagen had become mature and well organized. An occasional giant cell and some round cells could still be seen.

A review of the histological material indicated that the dynamics of the development of this membrane were, first, an acute inflammation followed by a cleaning up of the cellular elements and debris of this initial reaction, and, finally, organization which progressed from a predominantly fibrinous structure to a fairly mature fibrous membrane.

In Figure 2 can be seen the results of the penicillin assay of the serum and agar discs that were obtained when the agar discs for histological study were removed. Two to three determinations were made for each of the penicillin assays in order to contrast such variables as the degree of dicing of the agar and agitation of the tubes. The drug was administered two hours prior to removal of the discs and blood sample. It is seen that the penicillin content of the agar discs is in the same range throughout the 28 day experiment, with the exception of an unaccountably low concentration on Day 21. It is thus valid to assume that in this experimental situation penicillin was able to pass through the membrane surrounding the agar discs as well at 28 days as at any earlier time interval.

Rate of penicillin transport across membrane at 24 hours and 28 days. Information concerning the comparative rate at which penicillin entered the agar at 1 day and 28 days after placing the discs in the peritoneal cavity may be obtained from Figures 3 and 4 . At both times the discs and blood samples were removed at various intervals up to 24 hours after intravenous penicillin administration.

The results of the 24 hour experiment may be seen in Figure 3. For technical reasons, two animals were needed to complete the experiment. The serum concentrations fell off rapidly and were present in only negligible amounts after 2 hours, whereas the disc penicillin concentration 


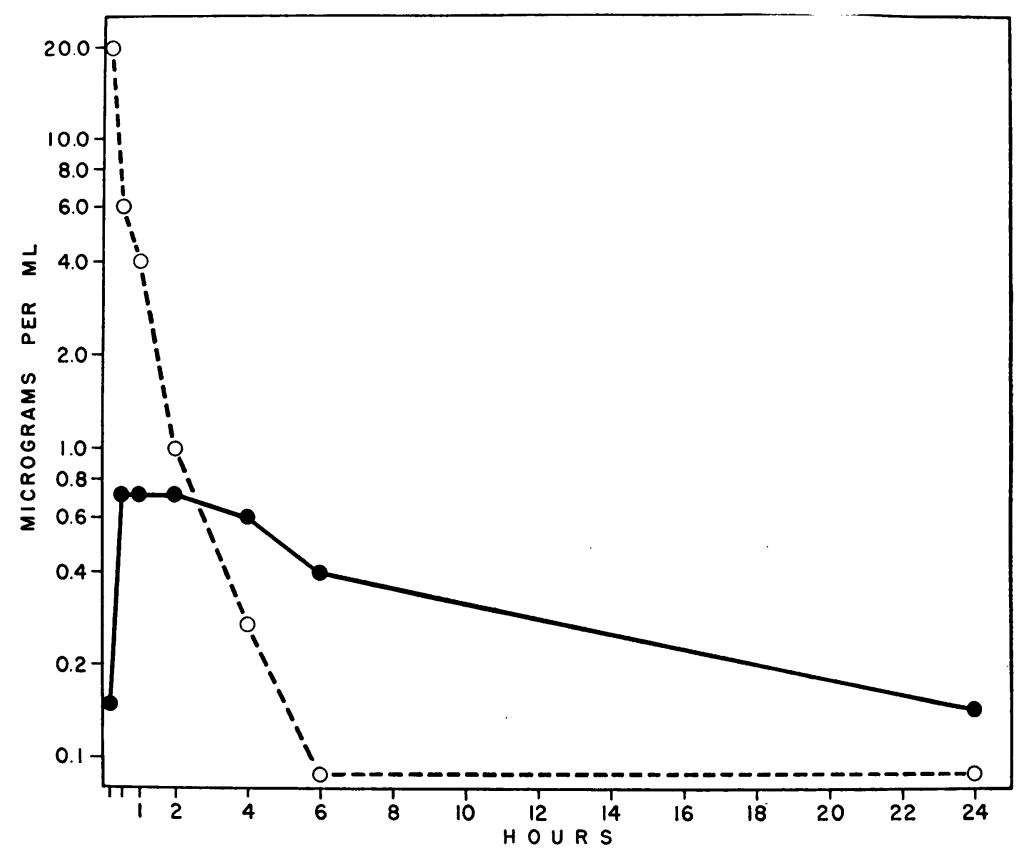

Fig. 4. Distribution of Penicillin Between Agar discs and Serum at 28 DAYS AFTER IMPLANTATION OF DISCS IN PERITONEAL CAVITY OF RABBITS. Penicillin injected two hours before removal of discs; $\bigcirc$ serum concentration; agar concentration.

was highest between 2 and 4 hours and began to fall off at 6 hours.

In Figure 4 can be seen the results of the companion experiment performed 28 days after in- serting the discs into the peritoneal cavity of another rabbit. It was again observed that the blood concentrations fell off rapidly whereas good agar concentrations, which were obtained early,
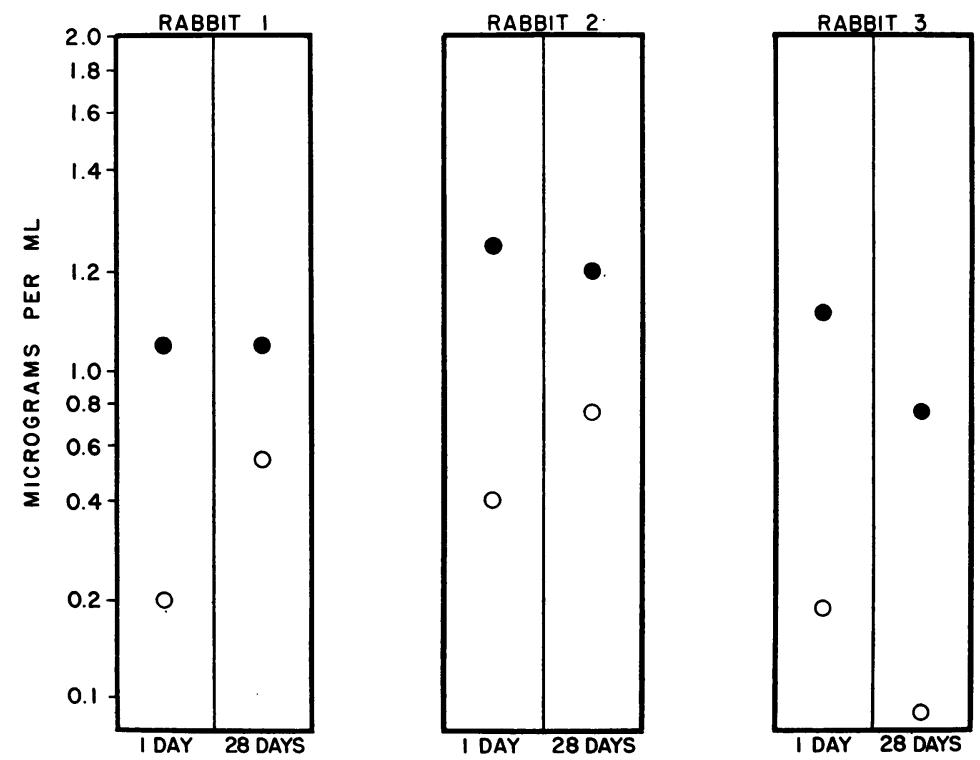

Fig. 5. Distribution OF PENICILlin BETWEen AGar disCS AND SERUM AT 1 DAY AND 28 DAYS AFTER IMPlantation OF DisCS IN PERITONEAL CAvity of RABBITs. Penicillin injected two hours before removal of discs; $\bigcirc$ serum concentration; agar concentration. 

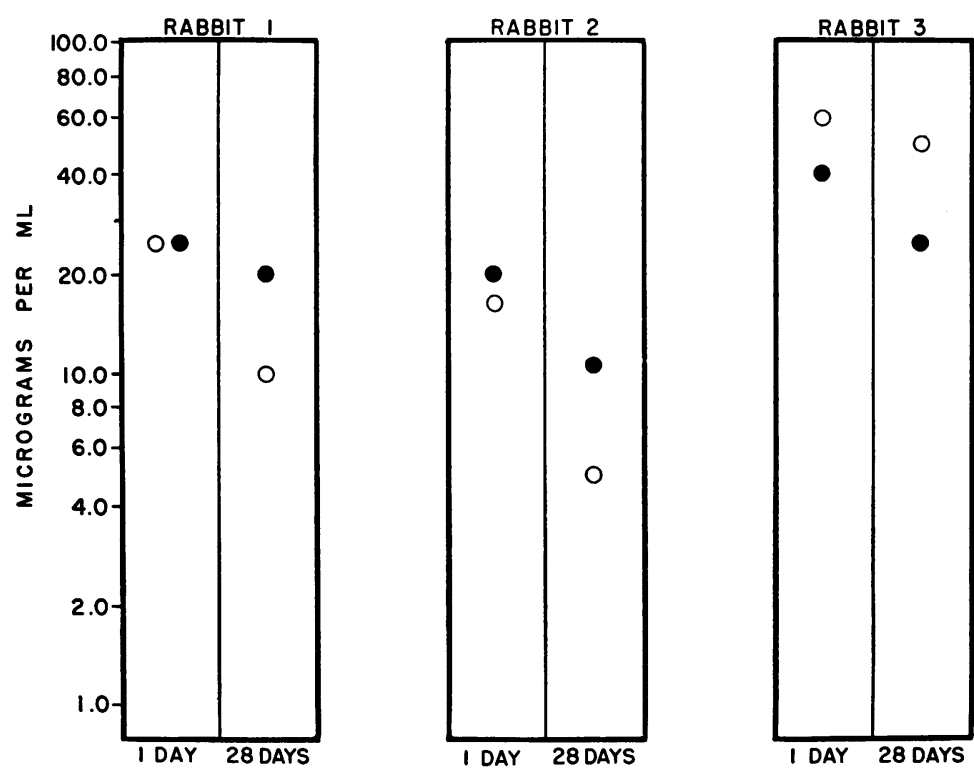

Fig. 6. Distribution OF STREPTOMycin BETWEen Agar Discs AND SERUM AT 1 DAY AND 28 DAYS AFTER IMPLANTATION OF DISCS IN PERITONEAL CAVITY of RABBITS. Streptomycin injected two hours before removal of discs; $\bigcirc$ serum concentration; agar concentration.

persisted for 6 hours. From these studies it appeared that, not only was penicillin able to pass through the membrane to the same degree, but also at the same rate at 28 days as at 24 hours.

In Figure 5 may be seen the results of the serum-agar penicillin concentration relationships at 24 hours and at 28 days after insertion of the agar discs into three animals. At each study point in all animals, the drug was administered 2 hours before removal of the blood sample and the agar discs. The results were similar in all animals and again demonstrated the equal ability of penicillin to penetrate the membrane at 28 days as at 24 hours.

Penetration of streptomycin at 24 hours and 28 days. An experimental model similar to that used in the experiment described above (Figure 5) may be seen in Figure 6 . In each of the three rabbits, blood samples and agar discs were removed 2 hours after the intravenous administration of streptomycin, $60 \mathrm{mg}$ per $\mathrm{kg}$. Determinations were conducted at 24 hours and at 28 days after implanting the agar discs intraperitoneally.

It is seen that comparable agar-serum streptomycin concentration relationships existed at 1 and at 28 days. There was a reasonable duplication of results in the three animals. It appears that streptomycin is as effective as penicillin in its ability to penetrate the membrane surrounding the agar disc 28 days after peritoneal implantation. The serum concentrations of streptomycin are seen to be higher than were the serum concentrations of penicillin (Figure 5), although they were measured at a comparable period of time after administration of the drug. This demonstrates that streptomycin is cleared more slowly from the blood than is penicillin.

\section{DISCUSSION}

The results of these studies indicated that penicillin and streptomycin were able to enter the substance of agar discs that had been inserted into the peritoneal cavity of rabbits. These discs were made of unenriched agar and, accordingly, as has been previously reported $(12,14)$, the subsistence of the experimentally implanted microorganisms depended entirely upon nutriment provided by the host. The present study has established that the distribution of these drugs into the agar occurred in an identical fashion whether they were administered immediately after implantation into the peritoneal cavity or 28 days thereafter, at which time a well organized fibrous membrane surrounded the agar disc. It was seen 
that the drug penetrated the agar disc at the same rate 24 hours or 28 days after peritoneal implantation, as is indicated in the penicillin studies.

The membrane about the agar disc developed in a manner comparable with that of an acute inflammatory process; there was an initial cellular reaction, followed by a gradual clearing of the cellular element and debris. Eventually, with the organization of the inflammatory reaction, the membrane matured from a predominantly fibrinous structure to a well developed fibrous process. The agar core, surrounded by the fibrous membrane, bore some resemblance to a "closed" tuberculous lesion with its core of solid caseous necrosis and its peripheral fibrous membrane.

Throughout the history of the development of antimicrobial drugs, the ability to penetrate some of the products of inflammation has rightly been considered a criterion of an effective therapeutic agent. Penicillin and streptomycin were the drugs employed in these studies because of their extensive use in the treatment of infections associated with the development of inflammatory barriers such as abscess walls, fibrinous vegetations, and other products of exudation and necrosis that potentially could impede the transport of a drug. As recently as 1951, abrasion of the lining of an abscess cavity was advocated because "substances such as penicillin probably do not penetrate granulation tissue" (15).

The observations herein reported confirm in vitro and in vivo studies of others $(8-12,14)$. They are likewise similar to results obtained by Florey, Turton and Duthie in the study of the influence of penicillin in wound infections after intramuscular administration (16). In this study the age of the wounds ranged from 4 to 32 days. Blood and exudate penicillin concentrations were similar at the end of the first hour after drug administration, but after this time the blood concentrations fell off more rapidly than did the exudate levels. In all cases there was a detectable amount of penicillin in the exudates up to 8 hours after injection, and in 50 per cent of the patients, up to 12 hours. It was observed that the results were similar irrespective of the age of the wound, the penicillin being recovered in comparable amounts and comparable periods of time after drug administration in 32-day-old wound exudates as in the younger lesions. Ran- dom studies of cavernostomy exudates and contents of tuberculous cavities from resected lungs have demonstrated substantial concentrations of streptomycin within the lesions (17).

The ability of drug-susceptible microorganisms to survive in mammalian tissues despite intensive antimicrobial therapy is a phenomenon that is poorly understood. The subject has been extensively reviewed by McDermott (18). This phenomenon of microbial persistence has been demonstrated in the laboratory in experimental animals with tuberculous infections (19) and in other studies concerned with staphylococcal disease (20). In both sets of studies a significant portion of the infecting microbial populations of tubercle bacilli or staphylococci was observed to persist in mouse tissues after prolonged therapy with the most effective antimicrobial substances available.

Among the possible explanations for this phenomenon are the emergence of drug-resistant organisms, the influence of the environmental characteristics of the inflammatory lesion, and factors of the drug dosage size. It could be conjectured that inflammatory barriers, such as the fibrous membranes surrounding the agar discs placed in the peritoneal cavity of rabbits described in this report, could support microbial persistence by impeding the penetration of the drug into the lesion. However, the transport of penicillin and streptomycin across this fibrous membrane occurred rapidly with both.

It is to be emphasized that the membrane utilized in these studies is probably not exactly like the inflammation seen in an actual infectious disease. It is fair to say, however, that the membrane represents the barrier by which a rabbit walls off an irritating substance in its peritoneal cavity, and that in its histological development it follows the classical anatomical course of inflammation and repair which is analogous to many of the inflammatory products associated with infectious processes.

\section{SUMMARY}

Placement of agar discs in the peritoneal cavity of rabbits was followed by development of a membrane which surrounded them. The nature of this tissue reaction followed the course of inflam- 
mation and repair and ultimately matured into a well organized fibrous membrane.

Penicillin and streptomycin were distributed into the discs equally as well after the development of the surrounding fibrous membrane as immediately after the intraperitoneal implantation of the discs. The rate of penetration of penicillin into the agar was similar whether or not the agar disc was surrounded by the fibrous membrane.

\section{ACKNOWLEDGMENT}

The technical assistance of Miss Carol Adams is gratefully acknowledged.

\section{REFERENCES}

1. Menkin, V. Dynamics of Inflammation. New York, Macmillan, 1940, p. 125.

2. Burrows, H. Some Factors in the Localization of Disease in the Body. Baltimore, Wm. Wood, 1932.

3. Fox, J. P. The localization and concentration of blood-borne antibodies and colloidal dye in areas of inflammation of various ages. J. Immunol. 1936, 31, 293.

4. Rigdon, R. H. Capillary permeability in the skin of the rabbit. Proc. Soc. exp. Biol. (N. Y.) 1939, 42, 43.

5. Markham, N. P., and Florey, H. W. The effect on experimental tuberculosis of the intravenous injection of insoluble substances: Experiments with carbon. Brit. J. exp. Path. 1951, 32, 25.

6. Hughes, W. H. The fibrin barrier regarded as a filter in inflammation. Brit. J. exp. Path. 1948, 29, 173.

7. Friedman, M. A study of the fibrin factor in its relation to subacute endocarditis. J. Pharmacol. exp. Ther. 1938, 63, 173.

8. Nathanson, M. H., and Liebhold, R. A. Diffusion of sulfonamides and penicillin into fibrin. Proc. Soc. exp. Biol. (N. Y.) 1946, 62, 83.
9. Mosonyi, L., Held, R., and Kocsan, C. On the diffusion of penicillin. Acta med. scand. 1949, 132, 487.

10. Weinstein, L., Daikos, G., and Perrin, T. S. Studies on the relationship of tissue fluid and blood levels of penicillin. J. Lab. clin. Med. 1951, 38, 712.

11. Ungar, J. Penicillin in tissue exudates after injection. Lancet 1950, 1, 56.

12. Werner, C. A., Knight, V., and McDermott, W. Studies of microbial populations artificially localized in vivo. I. Multiplication of bacteria and distribution of drugs in agar loci. J. clin. Invest. 1954, 33, 742.

13. Tompsett, R., Shultz, S., and McDermott, W. The relations of protein binding to the pharmacology and antibacterial activity of penicillins $\mathrm{X}, \mathrm{G}$, dihydro F, and K. J. Bact. 1947, 53, 581.

14. Darnell, J. E., Jr., Pesch, B. B., and Glaser, R. J. Effect of penicillin on group A streptococci in vivo in the absence of leukocytes. J. clin. Invest. $1955,34,1237$.

15. E1lis, M. The use of penicillin and sulphonamides in the treatment of suppuration. Lancet 1951, 1, 774.

16. Florey, M. E., Turton, E. C., and Duthie, E. S. Penicillin in wound exudates. Lancet 1946, 2, 405.

17. Canetti, G. Human lung tissue reactions to the tubercle bacillus in relation to chemotherapy. Ciba Found. Symp. exp. Tuberc., G. E. W. Wolstenholme and M. P. Cameron, Eds. Boston, Little, Brown, 1955, pp. 283-298.

18. McDermott, W. Microbial persistence. Yale J. Biol. Med. 1958, 30, 257.

19. McCune, R. M., Jr., Tompsett, R., and McDermott, W. The fate of Mycobacterium tuberculosis in mouse tissues as determined by the microbial enumeration technique. J. exp. Med. 1956, 104, 763.

20. McCune, R. M., Jr., Dineen, P. A. P., and Batten, J. C. The effects of antimicrobial drugs on an experimental staphylococcal infection in mice. Ann. N. Y. Acad. Sci. 1956, 65, 91. 